Anna Carlissa P. Arriola, MD

Thanh Vu T. De Guzman, MD

Department of Otorhinolaryngology

Head and Neck Surgery

Jose R. Reyes Memorial Medical Center
Correspondence: Dr. Anna Carlissa P. Arriola Department of Otorhinolaryngology Head and Neck Surgery 4th Floor, Jose R. Reyes Memorial Medical Center San Lazaro Compound, Rizal Avenue

Sta. Cruz, Manila 1003

Philippines

Phone: (632) 7436921 ; (632) 7119491 local 320

Email: carlyarriola@yahoo.com

Reprints will not be available from the author.

The authors declared that this represents original materia that is not being considered for publication or has not been published or accepted for publication elsewhere in full or in part, in print or electronic media; that the manuscript has been read and approved by all the authors, that the requirements for authorship have been met by each authors, and that each authorbelieves that the manuscript represents honest work.

Disclosures: The authors signed disclosures that there are no financial or other (including personal) relationships, intellectualpassion, political or religious beliefs, and institutional affiliations that might lead to a conflict of interest.

Presented at the Philippine Society of Otolaryngology Head and Neck Surgery, Free Paper Presentation, Sofitel Philippine Plaza, CCP Complex Roxas Boulevard, Pasay City, December 3, 2013

\section{Transcanal Resection of a Type 1 Glomus Tympanicum}

\begin{abstract}
Objectives: To present a case of type 1 glomus tympanicum, its clinical presentations, surgical management and outcome.
\end{abstract}

\section{Methods:}

Design: Case Report

Setting: Tertiary Government Hospital

Patient: One

Results: A 44-year-old woman with pulsatile tinnitus, vertigo, headache, ear fullness and decreased hearing on the right had a pulsatile reddish mass behind the tympanic membrane and Brown sign. Weber test lateralized to the right with mild conductive hearing loss on pure tone audiometry. Contrast CT scan demonstrated a $5 \times 6 \mathrm{~mm}$ welldefined enhancing mass in the meso- and hypotympanum. Internal auditory canal MRI showed an avidly enhancing $5 \times 3 \times 4 \mathrm{~mm}$ nodule within the right middle ear adjacent to the cochlear promontory and anterior to the lateral semicircular canal. Impression was glomus tympanicum, type 1 . The mass was excised via transcanal approach with postoperative resolution of tinnitus, headache, vertigo and improvement of hearing. Final histopathology was consistent with glomus tumor.

Conclusion: Glomus tympanicum tumors are rare, benign middle ear paragangliomas that arise from Jacobson's nerve are slow-growing and locally destructive. CT scan and MRI may detect involvement of other structures. Surgical resection is the primary treatment modality. Type 1 glomus tympanicum tumors are small and limited to the promontory and a less-invasive transcanal approach may be employed.

\section{Keywords: glomus, tympanicum, paraganglioma, transcanal approach}

Glomus tympanicum tumors are middle ear paragangliomas that arise from Jacobson's nerve. These are tumors of middle age, found two to five times more frequently in women than in men. ${ }^{1}$ They are benign, slow growing tumors which are locally destructive. ${ }^{2}$ Glomus tumors are rare with an estimated annual incidence of one case per 1.3 million people. ${ }^{3}$ Patients with these tumors present with pulsatile tinnitus and hearing loss. This case report will discuss a 44-yearold woman with glomus tympanicum type 1, highlight its manifestations, imaging, management and histopathologic correlates. 


\section{CASE REPORT}

A 44-year-old woman came to our outpatient department complaining of pulsatile tinnitus. Four months prior to consult, she started to have occasional pulsatile tinnitus on the right ear, the longest episode lasting 15 minutes. This was accompanied by vertigo described as movement of surroundings, lasting about 30 minutes which would spontaneously resolve. She noted the tinnitus to be accompanied by ipsilateral headache and right ear fullness. Headache was described as squeezing in character. Three months prior to consult, she noted decreased hearing of about $10 \%$ on the right. She had hypertension with highest blood pressure of 150/90 and took Losartan as maintenance medication. On review of systems, there were no palpitations, anxiety, diaphoresis, weight loss or flank pain.

On otoscopy, a pulsatile reddish mass was seen behind the tympanic membrane and Brown sign was elicited. (Figure 1) Weber test lateralized to the right ear where pure tone audiometry detected a mild conductive hearing loss with average of $30 \mathrm{~dB}$. Cranial nerve VII was intact. Blood pressure taken in supine and upright positions showed no significant difference. The systemic examination was normal. Our clinical impression was glomus tympanicum tumor.

Contrast-enhanced temporal bone CT (Figures 2 and 3) demonstrated a $5 \times 6 \mathrm{~mm}$ well-defined enhancing mass in the mesoand hypotympanum which slightly bulged the tympanic membrane. The ossicles were intact and there was no evidence of bony erosion. The vestibule, cochlea and semicircular canals had normal configuration. The carotid canal was intact and no high riding jugular vein or dehiscence was noted. No abnormalities were seen along course of the facial nerve canal. Magnetic resonance imaging of the internal auditory canal (Figures 4 and 5) showed an avidly enhancing nodule within the right middle ear measuring $5 \times 3 \times 4 \mathrm{~mm}$ in $A P$, transverse and craniocaudal dimensions. The nodule was adjacent to the cochlear promontory and anterior to the lateral semicircular canal. The seventh and eighth nerve complexes were normal. The cerebellopontine angles were unremarkable.

The patient underwent excision of the glomus tumor via a transcanal approach. Elevation of the tympanic membrane flap revealed a smooth, ovoid pinkish mass that covered approximately $50 \%$ of the middle ear. (Figure 6) The malleus, a portion of the incus and stapes and round window were still visible. The mass was bluntly dissected from the promontory. (Figure 7) Bleeding was controlled with epinephrinesoaked gelfoam. The flap was returned to its original position and the ear was packed with gelfoam and Ofloxacin otic drops. After the operation, the patient noted absence of tinnitus, headache and vertigo and improvement of hearing. Histopathologic examination showed small

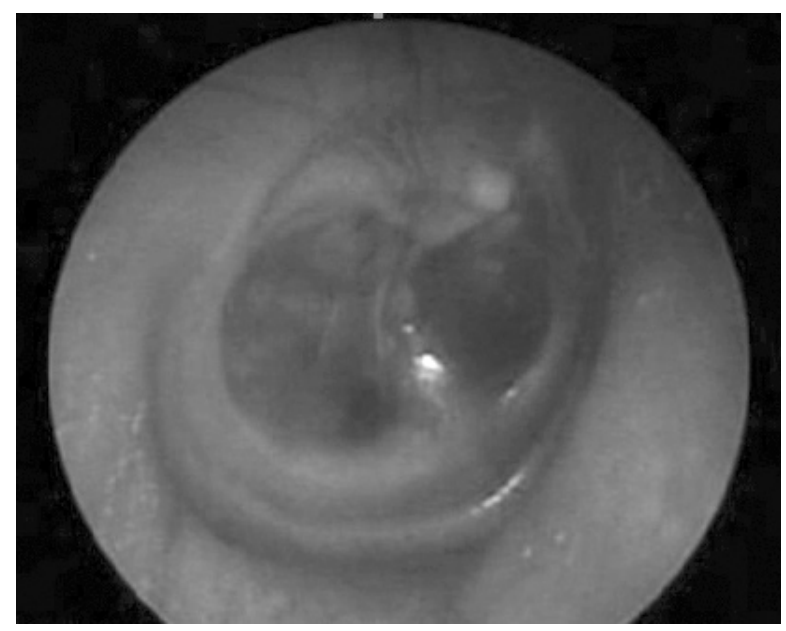

Figure 1. Pulsatile reddish mass behind the tympanic membrane

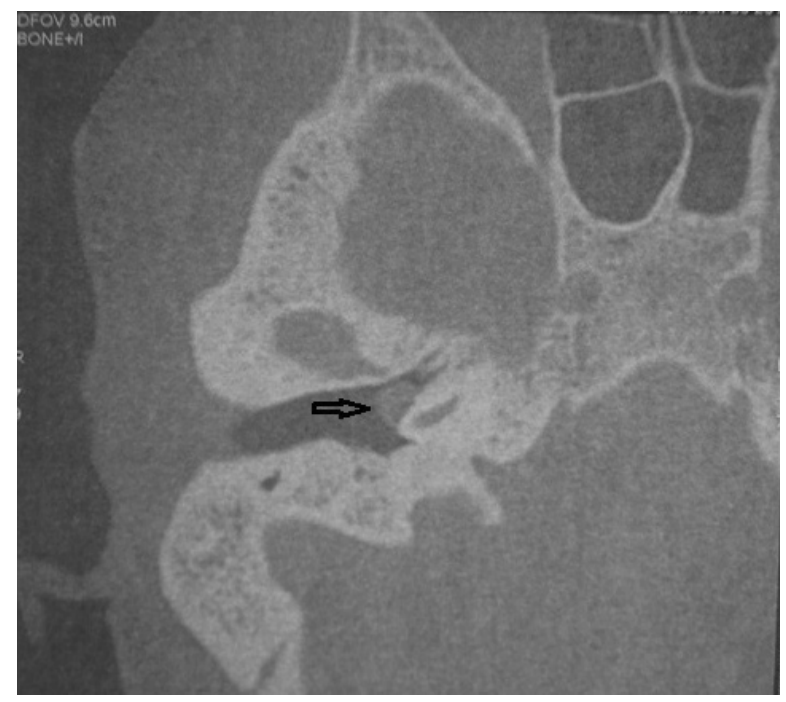

Figure 2. Contrast enhanced Temporal Bone CT Scan, Axial view, at the level of the external auditory canal, showing enhancing mass in the right middle ear.

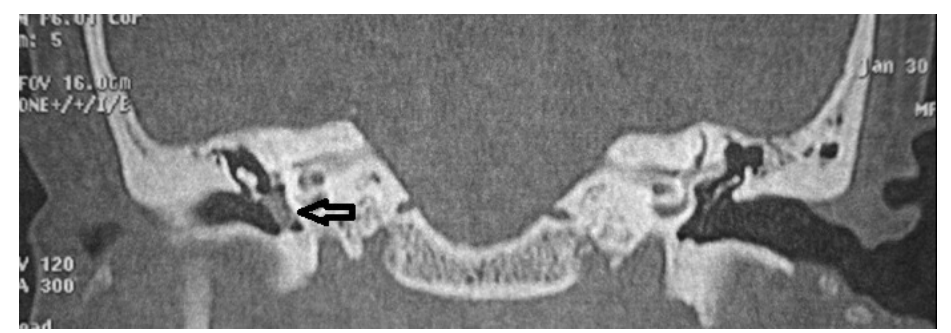

Figure 3. Contrast enhanced Temporal bone CT Scan, Coronal view, at the level of the external auditory canal, showing enhancing mass in the right meso and hypotympanum.

round cells infiltrating the entire stroma on low-power magnification, with prominent nuclei and eosinophilic cytoplasm on high-power magnification. (Figure 8) Final histopathology was consistent with glomus tumor. 


\section{CASE REPORTS}

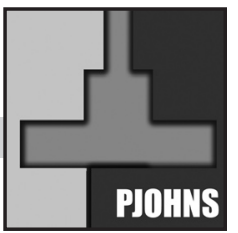

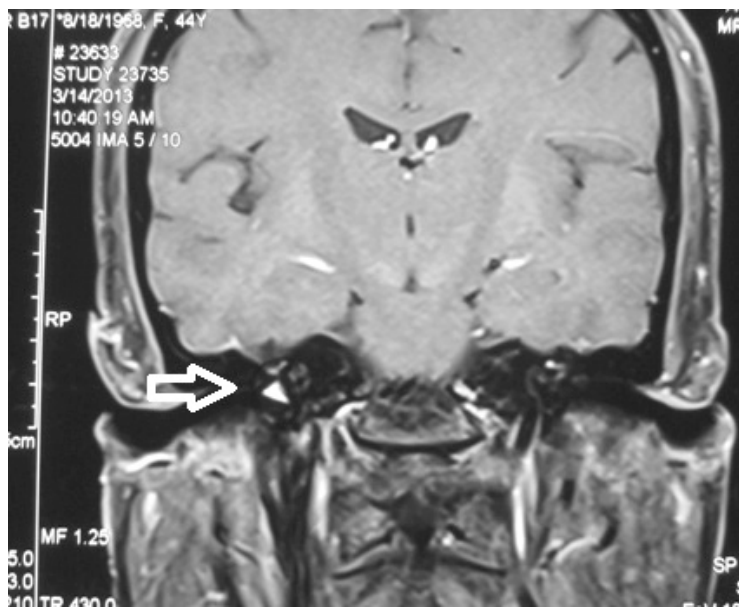

Figure 4. Gadolinium-DTPA enhanced T1-weighted Internal Auditory Canal MRI, Coronal view, showing enhancing nodule in the right middle ear.

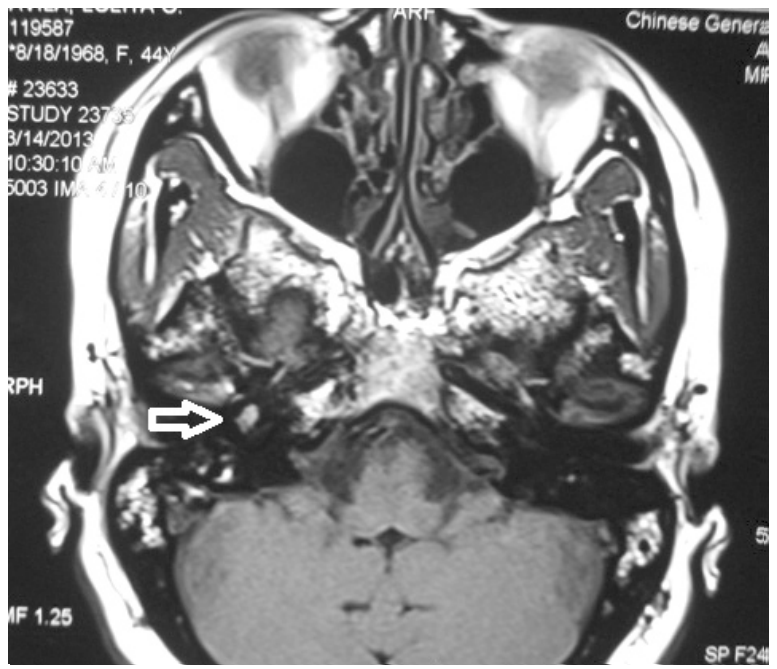

Figure 5. Gadolinium-DTPA enhanced T1-weighted Internal Auditory Canal MRI, Axial view, showing enhancing nodule in the right middle ear.

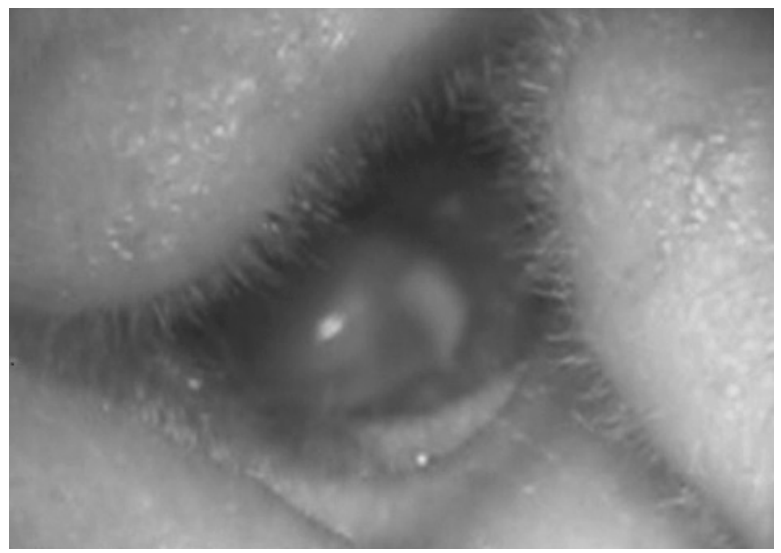

Figure 6. Pinkish ovoid mass in transcanal view

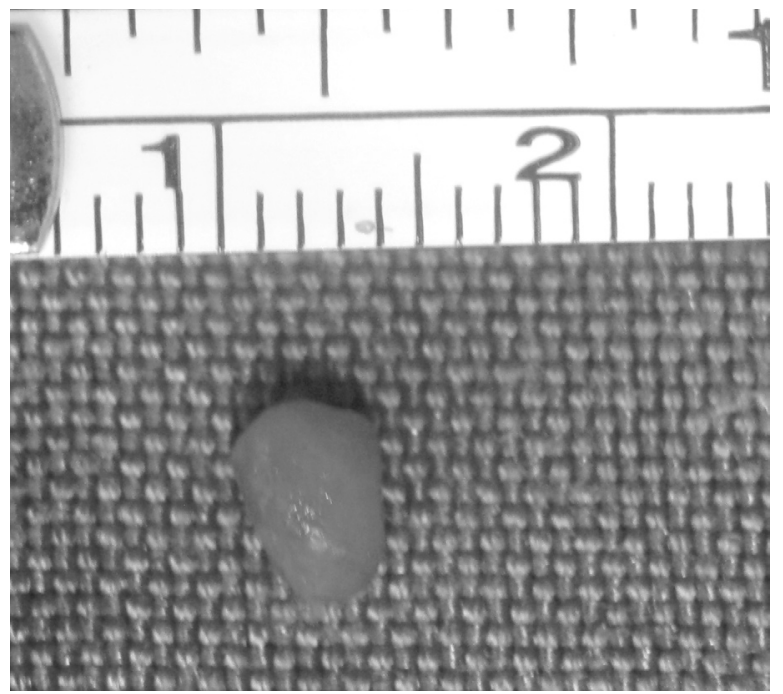

Figure 7. Excised Glomus

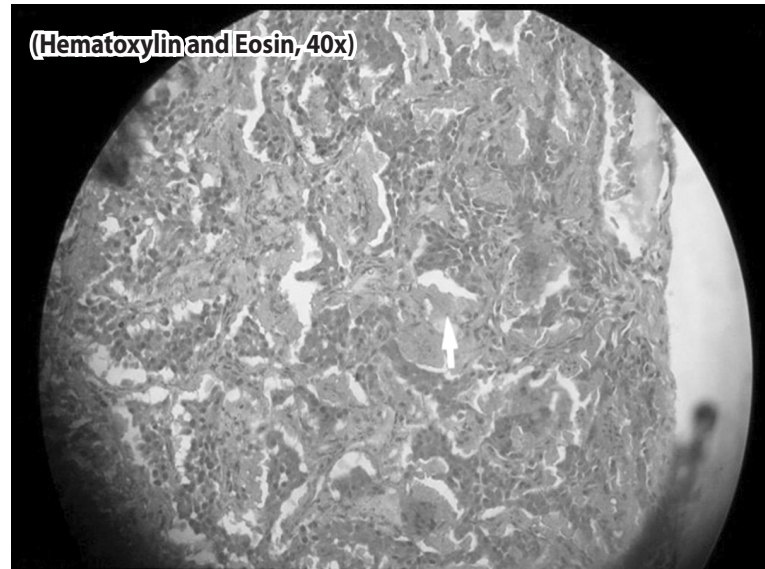

Figure 8. Hematoxylin and Eosin (40x) Round cells with eosinophilic cytoplasm surrounding blood vessels.

\section{DISCUSSION}

Paragangliomas are benign, slow growing tumors that arise from neuroectodermal tissues. Cervical paragangliomas and temporal bone (jugulotympanic) paragangliomas comprise paragangliomas of the head and neck. ${ }^{2}$ Paraganglia of the temporal bone are usually found in the mesotympanum and accompanying the inferior tympanic branch of the glossopharyngeal nerve. ${ }^{4}$ They serve as baroreceptors that sense and regulate oxygen pressure in the middle ear and mastoid cavity. ${ }^{5}$ These are well-vascularised lesions usually supplied by the inferior tympanic branch of the ascending pharyngeal artery. Paraganglions are composed of Type I (Zellballen) chief cells which are clusters of neural crest origin and are components of the diffuse neuroendocrine system, and Type II or sustentacular cells (modified Schwann cells), intimately 


\section{CASE REPORTS}

interlaced with a rich network of capillaries and venules. ${ }^{6}$

There are two commonly used classifications of glomus tympanicum tumors-- Oldring and Fisch and Glasscock and Jackson's. Glasscock and Jackson's system classifies glomus tympanicum by area and degree of involvement. Type 1 tumors are small and limited to the promontory, Type 2 tumors completely fill the middle ear. Type 3 tumors extend further into the mastoid while Type 4 tumors spread into the external auditory canal and may have intracranial extension. ${ }^{7}$ In this patient, the margins were visible 360 degrees around the circumference of the mesotympanic mass which qualified as a type 1 glomus tympanicum.

Patients with glomus tympanicum tumor present with pulsatile tinnitus and conductive hearing loss. Conductive hearing loss occurs when the tumor impairs normal vibration of the ossicles. Rarely, dizziness and sensorineural hearing loss may occur if the tumor has invaded the inner ear. ${ }^{2}$ This patient presented with pulsatile tinnitus and conductive hearing loss which was consistent with a glomus tympanicum. Vertigo and headache are red flags of tumor spread that may warrant further imaging. On CT and MRI, the glomus has not infiltrated the labyrinths. Every patient with a glomus tympanicum, except those with small type 1 glomus tumor should have serum catecholamine and urinary metabolite determination to rule out the probability of a functioning tumor. ${ }^{6}$ Functioning tumors may present with hypertension, tachycardia, orthostatic hypotension, excessive perspiration, tremor or vascular headaches. ${ }^{8}$ Although this patient had hypertension and headache, the size of the tumor and the absence of other signs and symptoms of a functioning tumor did not warrant catecholamine and urinary metabolite determination. On physical examination, the hallmark of a jugulotympanic glomus tumor is a reddish-blue mass seen behind the tympanic membrane. Brown sign described as the pulsation elicited by pneumatic compression that is abolished with further compression was present in the patient.

On CT scan, glomus tympanicum appears as a soft tissue mass abutting the promontory of the middle ear. ${ }^{9}$ Imaging with CT also allows visualization of ossicular displacement or bony erosion of the tympanic cavity and is best for evaluating bony destruction and erosion which is a hallmark of jugulotympanic glomus tumors. The patient initially had a CT scan but an MRI of the internal auditory canal was requested to visualize whether there was involvement of other structures such as major blood vessels. MRI is more advantageous than CT in delineating tumor edges and intracranial extent. It evaluates the relationship of the tumor to vascular structures such as the jugular vein and carotid artery and neck structures such as cranial nerves. ${ }^{10}$

The treatment of glomus tympanicum depends on the patient's age, site, size, extent of the tumor, rate of symptom progression, preoperative cranial nerve status, possibility of multicentricity, neurosecretory status and patient preference. ${ }^{11}$ Surgical resection is still considered the primary treatment modality. Depending on the type and extent of the tumor, surgical approaches based on the Glasscock-Jackson classification system include transmeatal, extended facial recess or canal wall-down mastoidectomy. ${ }^{12}$ The less invasive transcanal route may be employed for complete excision of type 1 glomus tympanicum tumors, as in our case.

\section{REFERENCES}

1. Barnes L, Peel RL, Verbin RS.Tumors of the nervous system. In: Barnes L, ed. Surgical Pathology of the Head and Neck. New York, NY: Marcel Dekker; 1985. p. 659-724.

2. O'Leary MJ, Shelton C, Giddings NA, Kwartler J, Brackmann DE. Glomus tympanicum tumours: a clinical perspective. Laryngoscope. 1991 Oct; 101(10): 1038-43.

3. Moffat DA, Hardy DG. Surgical management of large glomus jugulare tumours: infra- and transtemporal approach. J Laryngol Otol. 1989 Dec; 103(12):1167-80.

4. Rohit Jain Y, Caruso A, Russo A, Sanna M. Glomus tympanicum tumour: an alternative surgical technique. J Laryngol Otol. 2003 Jun; 117(6):462-6.

5. Manolidis S, Shohet JA, Jackson CG, Glasscock ME. Malignant glomus tumours. Laryngoscope. 1999 Jan; 109(1):30-4.

6. Glasscock ME, Shambaugh G. Surgery for Benign Tumors of the Temporal Bone. In: Surgery of the Ear USA: People's Medical Publishing House; 2010. p.730-739.

7. Jackson CG, Glasscock ME, Harris PF. Glomus tumours: Diagnosis, classification and management of large lesions. Otolaryngol. 1982 Jul; 08(7):401-6.

8. Schwaber MK, Glasscock ME, Jackson CG,_Nissen AJ, Smith PG. Diagnosis and management of catecholamine secreting tumors. Laryngoscope. 1984 Aug; 94(8):1008-15.

9. Noujaim SE, Pattekar MA. Paraganglioma of the temporal bone: role of magnetic resonance imaging versus computed tomography. Top Magn Reson Imaging. 2000; 11:108-22.

10. Vogl TJ, Mack MG, Juergens M, Bergman C, Grevers G, Jacobsen TL, et al. Skull base tumours gadodiamide injection-enhanced MR imaging. Radiology. 1993 Aug; 188(2):339-46.

11. Sen C, Hague K, Kacchara R, Jenkins A, Das S, Catalano P. Jugular foramen : microscopic anatomic features and implications for neural preservation with reference to glomus tumours involving the temporal bone. Neurosurgery. 2001Apr; 48(4): 838-47.

12. Forest JA 3rd, Jackson CG, McGrew BM. Long-term control of surgically treated glomus tympanicum tumours. Otol Neurotol. 2001 Mar; 22(2):232-6. 